\title{
Domínio Físico da Qualidade de Vida entre Adolescentes: Associação com Atividade Física e Sexo
}

\author{
Adolescents' physical quality of life: associations with \\ physical activity and sex
}

\author{
Alex Pinheiro Gordia ${ }^{1,2}$, Teresa M. Bianchini de Quadros ${ }^{2}$, Wagner de Campos ${ }^{1}$ y \\ Édio L. Petroski ${ }^{2}$ \\ 1 Centro de Pesquisa em Exercício e Esporte, Universidade Federal do Paraná, Curitiba, \\ alexgordia@gmail.com \\ 2 Núcleo de Pesquisa em Cineantropometria e Desempenho Humano, Universidade Federal de \\ Santa Catarina, Florianópolis, Santa Catarina, Brasil \\ Recebido em 11 Junho 2008/Enviado para Modificação 14 Dezembro 2008/Aprovado 11 Janeiro 2009
}

\section{RESUMO}

Objetivo Analisar as associações do Nível de Atividade Física (NAF), consumo de álcool, Índice de Massa Corporal (IMC) e variáveis sociodemográficas com o domínio físico da Qualidade de Vida (QV) de adolescentes.

Métodos A amostra foi composta por 608 adolescentes com idades entre 14 e 20 anos, de ambos os sexos, regularmente matriculados na rede pública e particular do ensino médio do município da Lapa, Paraná, Brasil. Foram investigadas variáveis referentes à QV dos adolescentes (domínio físico), NAF, consumo de álcool, IMC e variáveis sociodemográficas. Utilizou-se estatística descritiva, qui-quadrado, exato de fisher e regressão logística binária.

Resultados O domínio físico da QV dos adolescentes estudados apresentou resultados satisfatórios, com escore médio de 74,4. Indivíduos menos ativos tiveram 1,7 vezes mais risco para possuir domínio físico ruim do que seus pares mais ativos, bem como, moças tiveram 2,8 vezes mais chance de apresentar domínio físico ruim quando comparadas com rapazes.

Conclusão Os principais subgrupos populacionais com risco de apresentar domínio físico ruim foram adolescentes que apresentaram NAF baixo e pertencentes ao sexo feminino.

Palavras Chave: Qualidade de vida, atividade física, estilo de vida, comportamento do adolescente, gênero e saúde, Índice de Massa Corporal (fonte: DeCS, BIREME).

\section{ABSTRACT}

Objective Analysing associations between adolescents' physical activity level (PAL), alcohol consumption, body mass index (BMI) and sociodemographic variables and their physical quality of life (QoL).

Methods The sample consisted of 608 adolescents aged 14 to 20 who were fulltime 
students in public and private high schools in the municipality of Lapa, PR, southern Brazil. The variables analysed were the adolescents' physical QoL, PAL, alcohol consumption, BMI and sociodemographic variables. Descriptive statistics, the chi-square test, Fisher's exact test and binary logistical regression were used in the statistical analysis.

Results The physical QoL of the adolescents studied here exhibited satisfactory results (74.4 mean score). Less active individuals had 1.7 times greater risk of obtaining poor physical domain scores than their active counterparts; girls were 2.8 times more likely to have a poor physical domain score compared to boys.

Conclusions The adolescent population subsets most at risk of scoring badly on the physical domain were those having low PAL and being female.

Key Words: Quality of life, physical activity, lifestyle, adolescent behavior, gender and health, body mass index (source: MeSH, NLM).

\section{RESUMEN}

Dominio físico de la calidad de vida entre adolescentes: asociación con el sexo y la actividad física

Objetivo Analizar las asociaciones de nivel de actividad física (NAF), consumo de alcohol, índice de masa corporal (IMC) y las variables socio demográficas con el dominio físico de la calidad de vida (CV) de los adolescentes.

Métodos La muestra se conformó con 608 adolescentes de edades comprendidas entre los 14 y 20 años, de ambos sexos, matriculados en la red pública y red privada de la educación media del municipio de Lapa, Paraná, Brasil. Se investigaron las variables referentes a la CV de los adolescentes (dominio físico), NAF, el consumo de alcohol, el IMC y las variables socio demográficas. Se utilizó la estadística descriptiva, Chi-cuadrado, Test exacto de Fisher y regresión logística binaria.

Resultados El dominio físico de la CV de los adolescentes estudiados mostró resultados satisfactorios, con media de 74,4. Los individuos menos activos tuvieron 1,7 veces más riesgo de tener un dominio físico más pobre que sus pares activos; las niñas presentaron 2,8 veces más un dominio físico pobre que los varones.

Conclusión Los principales subgrupos poblacionales con riesgo de presentar dominio físico pobre fueron los adolescentes femeninos con el NAF bajo.

Palabras Clave: Calidad de vida, actividad física, estilo de vida, conducta del adolescente, género y salud, Índice de Massa Corporal (fuente: DeCS, BIREME).

$\mathrm{O}$ interesse atual pela temática Qualidade de Vida (QV) está relacionado à possibilidade de melhoria das condições de vida das pessoas através de ações pontuais em populações específicas, podendo envolver aspectos físicos, psicológicos, sociais e ambientais. Na área da saúde, diversos profissionais estão somando esforços com intuito de melhorar a QV dos indivíduos através de ações que visem proteger as pessoas de desfechos patológicos. Nesta perspectiva, destacam-se os aspectos físicos da QV (dor, fadiga, sono, atividades da vida coti- 
diana, dependência de medicação e capacidade de trabalho) por apresentarem relação ínfima com a incidência de doenças crônico-degenerativas $(1,2)$.

Estudos vêm demonstrando que mudanças no estilo de vida da sociedade contemporânea, tais como diminuição da atividade física, aumento do peso corporal e consumo exacerbado de bebidas alcoólicas, estão associadas às doenças crônico-degenerativas e QV pobre em adultos (3-5). Levando-se em consideração que fatores de risco para doenças crônicas podem ter seu início na adolescência, esta fase da vida pode ser considerada como chave para intervenções e modificações de hábitos e comportamentos, bem como melhora da QV. Entretanto, investigações que visam identificar a relação entre o estilo de vida e a QV de adolescentes ainda sãoescassas. Além disso, a maioria dos estudos utilizados focaliza somente adolescentes que possuem doenças crônicas, e desta forma, os aspectos relacionados à QV de adolescentes saudáveis ainda são pouco estudados.

Em adição, variáveis sociodemográficas têm se apresentado como determinantes do estilo de vida e QV adolescente, tais como: local de moradia, série escolar, turno de estudo e idade (6-8); sendo que estes fatores não podem ser negligenciados em estudos que visem investigar o comportamento e a QV de adolescentes.

Com base neste panorama, a presente investigação objetivou analisar as associações do Nível de Atividade Física (NAF), consumo de álcool, Índice de Massa Corporal (IMC) e variáveis sociodemográficas com o domínio físico da QV de adolescentes.

\section{MÉTODOS}

População e Amostra

O presente estudo foi desenvolvido com base no banco de dados do projeto de pesquisa "Análise da qualidade de vida, prevalência do consumo de álcool, nível de atividade física e índice de massa corporal em estudantes do ensino médio da cidade da Lapa-PR", realizado de junho a novembro de 2005. Os protocolos do estudo foram aprovados pelo Comitê de Ética em Pesquisa da Universidade Estadual de Ponta Grossa, Paraná, Processo nº 020/2005.

O projeto envolveu estudantes de 14 a 20 anos de idade, de ambos os sexos, regularmente matriculados na rede pública e particular do ensino médio do município da Lapa, no Estado do Paraná, Região Sul do Brasil. O município é considerado 
como integrante da região metropolitana de Curitiba, capital do estado. Apopulação do município, em 2005, foi estimada em 44733 habitantes. A Rede de Ensino contava com 1596 estudantes regularmente matriculados do $1^{\text {a }}$ ao $3^{\mathrm{a}}$ ano do ensino médio, distribuídos em sete escolas: duas da rede particular $(\mathrm{N}=75)$; duas públicas de área urbana $(\mathrm{N}=1$ 183) e três públicas de área rural $(\mathrm{N}=338)$. A perspectiva do estudo era avaliar todos os adolescentes matriculados no ensino médio. No entanto, a amostra final incluiu 608 sujeitos entre 14 e 20 anos de idade, que tiveram todos os dados tabulados e que participaram voluntariamente, representando $38,1 \%$ da população de estudantes do ensino médio, subdivididos nos seguintes estratos: Escolas Particulares, 24 adolescentes; Escolas Públicas da área Urbana, 391 adolescentes; Escolas Públicas da área Rural, 193 adolescentes, representando 32 \%, 33,1 \% e $57,1 \%$ de cada estrato, respectivamente. Desta forma, visando confirmar a representatividade da amostra investigada em relação à população de estudo, foi calculado o erro amostral para prevalência de sedentarismo de acordo com os critérios estabelecidos por Gil (9). O erro amostral encontrado (0,7 \%) foi inferior a $1 \%$, valor considerado excelente e que garante a representatividade da amostra analisada. Informações descritivas referentes à caracterização da amostra estudada podem ser observadas na Tabela 1.

Instrumentos e Procedimentos

Primeiramente, foi enviado aos pais e/ou responsáveis dos adolescentes o Termo de Consentimento Livre e Esclarecido (TCLE). Com a devolução do TCLE devidamente assinado, o adolescente era incluído na amostra para a subsequiente avaliação. Foram investigadas variáveis referentes à QV dos adolescentes (domínio físico), NAF, consumo deálcool, IMC e variáveis sociodemográficas (tipo de escola, local de moradia, sexo, condição socioeconômica, idade, turno de estudo e série escolar).

A QV foi mensurada através do questionário WHOQOL-Bref, desenvolvido pelo grupo de estudos sobre QV da OMS (10). Este instrumento contém 26 questões e considera os últimos quinze dias vividos pelos respondentes. O WHOQOL-Bref é composto por quatro domínios: físico, psicológico, relações sociais e meio ambiente. Entretanto, para o presente estudo foram utilizadas apenas informações referentes ao domínio físico, tais como, dor e desconforto, energia e fadiga, sono e repouso, mobilidade, atividades da vida cotidiana, dependência de medicação ou de tratamentos e capacidade de trabalho. Fleck et al. (11), demonstraram que a versão abreviada em português no Brasil do WHOQOL-Bref apresenta características satisfatórias de consistência interna, validade discriminante, validade de critério, validade concorrente e fidedignidade teste-reteste. Para adolescentes, tem sido 
relatado que o instrumento possui um conteúdo válido e propriedades psicométricas aceitáveis para mensurar a QV (12).

Tabela 1. Caracterização da amostra com valores expressos em média e desvio padrão

\begin{tabular}{|c|c|c|c|c|}
\hline & $N$ & Idade (anos) & $\begin{array}{c}\text { Massa } \\
\text { corporal }(\mathrm{kg})\end{array}$ & $\begin{array}{c}\text { Estatura } \\
(\mathrm{m})\end{array}$ \\
\hline \multicolumn{5}{|l|}{ Tipo de escola } \\
\hline Pública & 584 & $15,88(1,25)$ & $58,23(10,06)$ & $\begin{array}{c}1,66 \\
(0.09)\end{array}$ \\
\hline Particular & 24 & $15,21(0,93)$ & $63,06(15,92)$ & $\begin{array}{l}1,67 \\
(0,08)\end{array}$ \\
\hline \multicolumn{5}{|l|}{$\begin{array}{l}\text { Local de } \\
\text { moradia }\end{array}$} \\
\hline Área urbana & 415 & $15,87(1,21)$ & $58,18(10,72)$ & $\begin{array}{c}1,66 \\
(0.09)\end{array}$ \\
\hline Área rural & 193 & $15,82(1,32)$ & $58,93(9,63)$ & $\begin{array}{l}1,67 \\
(0,09)\end{array}$ \\
\hline \multicolumn{5}{|l|}{ Sexo } \\
\hline Masculino & 239 & $16,01(1,33)$ & $62,69(11,04)$ & $\begin{array}{c}1,73 \\
(0.08)\end{array}$ \\
\hline Feminino & 369 & $15,76(1,18)$ & $55,65(8,92)$ & $\begin{array}{c}1,62 \\
(0,07)\end{array}$ \\
\hline \multicolumn{5}{|l|}{$\operatorname{CS}$} \\
\hline A & 31 & $15,74(1,26)$ & $67,15(17,37)$ & $\begin{array}{c}1,70 \\
(0,09)\end{array}$ \\
\hline B & 163 & $15,74(1,12)$ & $58,37(10,23)$ & $\begin{array}{c}1,67 \\
(0,09)\end{array}$ \\
\hline C & 279 & $15,93(1,26)$ & $58,30(9,45)$ & $\begin{array}{c}1,66 \\
(0,09)\end{array}$ \\
\hline D & 128 & $15,92(1,37)$ & $56,79(9,42)$ & $\begin{array}{c}1,64 \\
(0,08)\end{array}$ \\
\hline E & 7 & $15,00(0,82)$ & $55,50(9,47)$ & $\begin{array}{c}1,61 \\
(0,08)\end{array}$ \\
\hline $\begin{array}{l}\text { Série escolar } \\
1^{\circ} \text { ano }\end{array}$ & 256 & $15,07(1,01)$ & $56,55(9,58)$ & 1,65 \\
\hline $2^{\circ}$ ano & 219 & $16,12(1,09)$ & $59,52(9,76)$ & $\begin{array}{c}(1,67) \\
(0.09)\end{array}$ \\
\hline $3^{\circ}$ ano & 133 & $16,93(0,84)$ & $60,20(12,21)$ & $\begin{array}{l}1,68 \\
(0,09)\end{array}$ \\
\hline \multicolumn{5}{|c|}{ Turno de estudo } \\
\hline Matutino & 302 & $15,74(1,21)$ & $59,44(10,79)$ & $\begin{array}{c}1,67 \\
(0,09)\end{array}$ \\
\hline Vespertino & 203 & $15,82(1,22)$ & $56,82(9,53)$ & $\begin{array}{c}1,65 \\
(0,08)\end{array}$ \\
\hline Noturno & 103 & $16,27(1,32)$ & $58,57(10,48)$ & $\begin{array}{c}1,66 \\
(0,10)\end{array}$ \\
\hline Amostra total & 608 & $15,86(1,24)$ & $58,42(10,38)$ & $\begin{array}{c}1,66 \\
(0.09)\end{array}$ \\
\hline
\end{tabular}

O WHOQOL-Bref foi analisado com base nos critérios propostos pela equipe australiana do WHOQOL (13), com resultados variando em uma escala de 0 a 100 , ressalvando que quanto mais próximo de 100 melhor é a QV do avaliado. Para que fosse possível verificar a distribuição dos adolescentes estudados em relação ao domínio físico foi realizada uma classificação mediante intervalo quartilar. 
O NAF foi mensurado através do Questionário Internacional de Atividade Física (International Physical Activity Questionnaire-IPAQ; versão 8, forma curta, última semana), validado para adolescentes (14). Para classificar o NAF, foi utilizada a técnica de categorização da amostra por meio de quartis, tendo em vista o poder das análises estatísticas.

O consumo de álcool foi mensurado através do Teste para a Identificação de Problemas Decorrentes do Uso de Álcool (AUDIT), instrumento desenvolvido pela OMS. Lima et al. (15) realizaram um estudo de validade concorrente e de construto do AUDIT para utilização em estudos epidemiológicos com a população brasileira, e os resultados indicaram alta consistência interna $(\alpha=0.81)$, sensibilidade de $100 \%$ e especificidade de $78 \%$, demonstrando sua validade para utilização em estudos epidemiológicos. O consumo de álcool foi analisado com base nas recomendações de Babor et al. (16), sendo que, de acordo com a soma dos escores do questionário, os adolescentes foram classificados como: abstêmios, consumidores de baixo risco, consumidores de risco, consumidores de alto risco e prováveis dependentes.

As medidas do peso corporal e estatura foram mensuradas de acordo com procedimentos e técnicas padronizadas (17), sendo utilizadas para o cálculo do IMC (kg.m $\left.{ }^{-2}\right)$. Para a classificação do IMC entre os adolescentes de 14 a 19 anos de idade, aplicou-se o critério proposto por Conde e Monteiro (18). Para classificação do IMC dos adolescentes com 20 anos de idade, utilizou-se os pontos de corte estabelecidos pela OMS (19).

A condição socioeconômica foi avaliada de acordo como Critério de Classificação Econômica do Brasil, desenvolvido pela Associação Brasileira de Empresas de Pesquisa (20). Para este estudo, as classes socioeconômicas foram agrupadas em classe alta $(\mathrm{A} 1+\mathrm{A} 2)$, classe média $(\mathrm{B} 1+\mathrm{B} 2)$ e classe baixa $(\mathrm{C}+\mathrm{D}+\mathrm{E})$, com intuito estatístico.

Análise estatística

Inicialmente, recorreu-se a análise descritiva do domínio físico da QV dos adolescentes estudados. Em seguida, utilizou-se o teste do Qui-quadrado, e quando necessário o teste exato de Fisher, para investigar diferenças proporcionais. A regressão logística binária (análise bruta e ajustada) foi usada para examinar as associações entre NAF, consumo de álcool, IMC e variáveis sociodemográficas (variáveis independentes) com o domínio físico (variável dependente) dos adolescentes. Todas as variáveis investigadas cujas associações com o domínio físico 
apresentaram valores de $\mathrm{p}<0,25$ no teste qui-quadrado, foram introduzidas no modelo de regressão, uma a uma, de maneira crescente, conforme sua significância estatística e/ou que foram consideradas relevantes de acordo com a literatura. Para a análise de regressão, os indivíduos pertencentes ao primeiro e segundo quartil foram classificados como domínio físico ruim e os adolescentes pertencentes ao terceiro e quarto quartil foram classificados como domínio físico bom. O nível de significância foi estabelecido em $\mathrm{p}<0,05$.

\section{RESULTADOS}

Com base na análise do questionário WHOQOL-Bref, observou-se escore médio para o domínio físico de 74,4, com desvio padrão de 12. Quando levado em consideração a QV global dos adolescentes (média de todos os domínios), foi encontrado escore médio de 67,3, com desvio padrão de 10,2.

A Tabela 2 apresenta uma análise associativa preliminar realizada para verificar a distribuição dos adolescentes em relação ao domínio físico, levando em consideração aspectos sociodemográficos, com estratificação por tipo de escola, local de moradia, sexo, condição socioeconômica e idade. Os achados demonstraram que apenas na estratificação por sexo foram encontradas diferenças significativas, sendo que, os rapazes apresentaram melhores resultados em relação às moças $\left(\mathrm{X}^{2}=43,18 ; \mathrm{p}=0,001\right)$.

No que diz respeito à análise de regressão logística binária bruta para variáveis associadas ao domínio físico (Tabela 3), obteve-se resultado significativo apenas para atividade física, sexo e série escolar. Adolescentes menos ativos $\left(1^{\circ}\right.$ e $2^{\circ}$ quartil) apresentaram mais chance de possuir domínio físico ruim quando comparados com adolescentes mais ativos, pertencentes ao $4^{\circ}$ quartil. Quanto ao sexo, moças tiveram 2,6 vezes mais chance de possuir domínio físico ruim em relação aos rapazes. Para a série escolar, adolescentes do $1^{\circ}$ ano do ensino médio apresentaram 1,6 vezes mais chance de possuir domínio físico ruim do que estudantes do $3^{\circ}$ ano.

Quando a análise foi ajustada por fatores sociodemográficos e demais variáveis independentes do modelo (Tabela 3), apenas a atividade física e o sexo permaneceram significativos, sendo que indivíduos menos ativos, pertencentes ao $2^{\circ}$ quartil, foram 1,7 vezes mais prováveis para possuir domínio físico ruim do que seus pares mais ativos, bem como, moças tiveram 2,8 vezes mais chance de apresentar domínio físico ruim quando comparadas com rapazes. 
Tabela 2. Classificação do domínio físico dos adolescentes através da distribuição quartilar de acordo com tipo de escola, local de moradia, sexo, condição socioeconômica e idade

\begin{tabular}{|c|c|c|c|c|}
\hline \multirow{2}{*}{$\begin{array}{l}\text { Variáveis } \\
\text { sociode mográficas }\end{array}$} & \multicolumn{4}{|c|}{ Classificação do Domínio Físico (\%) } \\
\hline & $\begin{array}{c}1^{\circ} \\
\text { quartil }\end{array}$ & $\begin{array}{c}2^{\circ} \\
\text { quartil }\end{array}$ & $\begin{array}{c}3^{\circ} \\
\text { quartil }\end{array}$ & $\begin{array}{c}4^{\circ} \\
\text { quartil }\end{array}$ \\
\hline \multicolumn{5}{|l|}{ Tipo de Escola } \\
\hline Pública & 22,2 & 23,4 & 33,2 & 21,4 \\
\hline Particular & 20,8 & 29,2 & 25,0 & 25,0 \\
\hline \multicolumn{5}{|l|}{ Local de Moradia } \\
\hline Área Rural & 18,1 & 21,8 & 34,7 & 25,4 \\
\hline Área Urbana & 23,8 & 24,5 & 32,0 & 19,7 \\
\hline \multicolumn{5}{|l|}{ Sexo } \\
\hline Masculino & 11,7 & 20,1 & 36,0 & 32,2 \\
\hline Feminino & 28,6 & 25,9 & 30,8 & 14,7 \\
\hline \multicolumn{5}{|l|}{ Condição } \\
\hline \multicolumn{5}{|l|}{ Socioeconômica } \\
\hline $\begin{array}{l}A \\
B\end{array}$ & 22,6 & $\begin{array}{c}6,4 \\
252\end{array}$ & $\begin{array}{l}45,2 \\
319\end{array}$ & 25,8 \\
\hline$C, D$ e E & 21,7 & 24,2 & 32,4 & 21,7 \\
\hline \multicolumn{5}{|l|}{ Idade } \\
\hline 14 & 20,5 & 27,5 & 31,5 & 20,5 \\
\hline 15 & 23,4 & 23,4 & 32,5 & 20,7 \\
\hline 16 & 22,2 & 21,1 & 35,1 & 21,6 \\
\hline 17 & 20,8 & 23,7 & 32,7 & 22,8 \\
\hline 18 & 20,5 & 29,5 & 27,3 & 22,7 \\
\hline 19 & 182 & 182 & 363 & 27,3 \\
\hline 20 & 33,3 & 167 & 33,3 & 16.7 \\
\hline
\end{tabular}

\section{DISCUSSÃO}

Os resultados descritivos do domínio físico da QV indicaram escore médio alto, demonstrando que este domínio apresentou-se como aspecto positivo da QV dos jovens pesquisados. Este achado pode ser explicado pelas facetas que englobam este domínio (dor e desconforto, energia e fadiga, sono e repouso, mobilidade, atividades da vida cotidiana, dependência de medicação ou de tratamentos e capacidade de trabalho), tendo em vista que adolescentes, em geral, são saudáveis e não apresentam limitações físicas. Outros estudos também reportam que o domínio físico é um forte contribuinte para QV positiva entre jovens $(21,22)$.

Os achados referentes à distribuição dos adolescentes em relação ao domínio físico, após estratificação por variáveis sociodemográficas, demonstraram que adolescentes do sexo masculino apresentaram QV melhor quando comparados com o sexo feminino. Este fato pode estar relacionado a diferenças nas condições de vida ou a diferenças na percepção da QV. No entanto, tendo em vista que outros estudos, realizados com jovens e adultos, também vêm observando que homens possuem QV melhor do que mulheres (23-24), pode-se supor que a principal diferença na avaliação da QV esteja na maior exigência das adolescentes do sexo feminino em relação à percepção da QV, ou seja, rapazes e moças podem ter condições de vida semelhantes, porém, formas diferentes para analisar e ponderar diversos aspectos de sua vida. 
Tabela 3. Associação entre o domínio físico da QV com a atividade física, consumo de álcool, IMC e variáveis sociodemográficas em adolescentes

\begin{tabular}{|c|c|c|c|}
\hline $\begin{array}{l}\text { Variáveis } \\
\text { (Categorias) }\end{array}$ & $\begin{array}{c}\mathrm{DF}^{\#} \\
\text { Ruim } \\
(\%)\end{array}$ & $\begin{array}{l}\text { Análise bruta } \\
\text { RC }(95 \% \text { IC) }\end{array}$ & $\begin{array}{c}\text { Análise } \\
\text { ajustada***} \\
\text { RC }(95 \% \text { IC })\end{array}$ \\
\hline \multicolumn{4}{|l|}{ Atividade Física } \\
\hline $4^{\circ}$ quartil & 36,8 & $1^{*}$ & $1^{* *}$ \\
\hline $3^{\circ}$ quartil & 43,4 & $1,31(0,83-2,08)$ & $\begin{array}{c}1,21(0,75- \\
1,96)\end{array}$ \\
\hline $2^{\circ}$ quartil & 52,0 & $1,86(1,17-2,93)$ & $\begin{array}{c}1,70(1,04- \\
2,77)\end{array}$ \\
\hline $1^{\circ}$ quartil & 50,0 & $1,71(1,08-2,71)$ & $\begin{array}{c}1,58(0,98- \\
2,57)\end{array}$ \\
\hline \multicolumn{4}{|l|}{ Consumo de álcool } \\
\hline Abstêmios & 43,0 & 1 & 1 \\
\hline Baixo Risco & 46,8 & $1,17(0,72-1,90)$ & $\begin{array}{c}1,20(0,71- \\
2,03)\end{array}$ \\
\hline Risco & 41,5 & $0,94(0,52-1,69)$ & $\begin{array}{c}1,16(0,61- \\
2,18)\end{array}$ \\
\hline Alto Risco & 39,3 & $0,86(0,36-2,06)$ & $\begin{array}{c}1,27(0,49- \\
3,31)\end{array}$ \\
\hline $\begin{array}{l}\text { Provável } \\
\text { Dependente }\end{array}$ & 66,7 & $2,65(0,83-8,46)$ & $\begin{array}{c}2,78(0,79- \\
9,78)\end{array}$ \\
\hline \multicolumn{4}{|l|}{$\begin{array}{l}\text { Índice de Massa } \\
\text { Corporal }\end{array}$} \\
\hline Peso Normal & 44,7 & 1 & 1 \\
\hline Baixo Peso & 50,0 & $1,24(0,43-3,58)$ & $\begin{array}{c}0,84(0,27- \\
2,56)\end{array}$ \\
\hline Sobrepeso & 50,7 & $1,28(0,77-2,13)$ & $\begin{array}{c}1,31(0,76- \\
2,27)\end{array}$ \\
\hline Obeso & 50,0 & $1,24(0,39-3,89)$ & $\begin{array}{c}2,01(0,55- \\
7,30)\end{array}$ \\
\hline \multicolumn{4}{|l|}{ Sexo } \\
\hline Masculino & 31,8 & $1^{*}$ & $1^{* *}$ \\
\hline Feminino & 54,5 & $2,57(1,83-3,61)$ & $\begin{array}{c}2,75(1,94- \\
3,91)\end{array}$ \\
\hline $\begin{array}{l}\text { Série Escolar } \\
1^{\circ}\end{array}$ & 51,6 & $1,56(1,02-2,38)$ & $\begin{array}{c}1,55(0,99- \\
2,42)\end{array}$ \\
\hline $2^{\circ}$ & 41,6 & $1,04(0,67-1,61)$ & $\begin{array}{c}1,06(0,67- \\
1,67)\end{array}$ \\
\hline $3^{\circ}$ & 40,6 & $1^{*}$ & 1 \\
\hline
\end{tabular}

* pd $\leq 0,05$ para a análise bruta; ${ }^{* *}$ pd $\leq 0,05$ para a análise ajustada; *** Análise ajustada para 0 tipo de escola, local de moradia, condição socioeconômica, idade, turno de estudo e para as demais variáveis independentes do modelo de regressão; \#DF=Domínio Físico

Quanto aos resultados da regressão logística, observou-se que após controle por fatores sociodemográficos e demais variáveis independentes do modelo, apenas a atividade física e o sexo apresentaram associação significativa com o domínio físico. Parece que adolescentes do sexo feminino são mais exigentes em relação à percepção de sua QV do que seus pares do sexo masculino. No entanto, outros estudos são necessários para confirmar estes achados, em especial, pesquisas com enfoque qualitativo referente à forma como os adolescentes percebem e avaliam sua $\mathrm{QV}$. 
O achado mais relevante da análise de regressão foi que indivíduos menos ativos foram mais prováveis para possuir domínio físico ruim do que seus pares mais ativos. A relação entre o domínio físico e a atividade física era esperada, tendo em vista evidências empíricas de que a prática de atividade física auxilia nas facetas que compõem este domínio. Estudo realizado por Chen et al. (25) objetivou examinar a extensão das mudanças no estilo de vida no final da infância e início da adolescência durante um período de acompanhamento de três anos, bem como analisar as associações entre hábitos de vida e QV. Os achados demonstraram que durante a transição da infância para o início da adolescência ocorreram consideráveis mudanças dos hábitos de vida, sendo que o NAF baixo na infância foi diretamente relacionado à $\mathrm{QV}$ ruim na adolescência. Em pesquisa semelhante, Wang et al. (26), investigaram o papel do estilo de vida durante a infância sobre a QV na adolescência. Os autores observaram que diversos comportamentos modificáveis durante a infância interferiram negativamente na QV de adolescentes, tais como, consumo alimentar inadequado, dormir pouco e inatividade física.

Os resultados do presente estudo, embasados por demais pesquisas, suportam que a prática regular da atividade física pode auxiliar na melhoria da $\mathrm{QV}$ de adolescentes, principalmente em relação ao domínio físico. Estes achados são de suma importância por estabelecer uma relação concreta entre a atividade física e a QV de adolescentes, podendo servir como suporte para que projetos de saúde pública considerem a atividade física como variável indispensável durante o desenvolvimento de programas de prevenção e intervenção.

Com base nos achados referentes ao domínio físico da QV dos adolescentes investigados, algumas alternativas que visem melhorar as condições de vida desta população podem ser desenhadas dentro do próprio ambiente escolar por se tratar de um meio propício para intervenções. Neste sentido, sugere-se o incentivo e auxilio a prática de todos os tipos de atividade física, visando o aumento do NAF entre adolescentes. Esta tarefa é, em especial, destinada aos professores de Educação Física, tendo em vista sua participação ativa na vida destes jovens dentro da escola.

Evidentemente, torna-se limitado avaliar exclusivamente por meio de quantificações um conceito intrinsecamente marcado pela subjetividade, como o construto QV. É preciso ter em mente que os indicadores e índices medem sempre "aspectos" da QV, tendo méritos e limitações uns em relação aos outros. Neste sentido, os resultados observados no presente estudo precisam ser analisados com cautela, tendo em vista que a medida "objetiva" da QV foi mensurada de forma subjetiva através de questionário. No entanto, é importante ressaltar que o instrumento utilizado tem se apresentado como uma possibilidade de análise da QV prática e confiável; emadição, o WHOQOL-Bref é recomendado pela OMS para avaliação da QV (10). 
Além da dificuldade para mensurar a QV, outra limitação do presente estudo consiste no fato de que a atividade física foi avaliada por meio de questionário, ao invés de métodos diretos como, por exemplo, acelerômetros. Desta forma, pode ter ocorrido subestimação ou superestimação do NAF dos adolescentes investigados. Entretanto, questionários são métodos atrativos em grandes amostras devido ao baixo custo e rapidez na obtenção dos dados. Em adição, o IPAQ é utilizado internacionalmente para avaliar a atividade física, e tem vantagens em comparação a outros instrumentos, principalmente no que se refere à possibilidade de comparação com outras pesquisas (27).

O presente estudo contribuiu para o progresso científico da área, em especial, por apresentar dados inéditos referentes a variáveis determinantes de aspectos físicos da QV adolescente que podem ser utilizados em programas de saúde pública. Cabe salientar que a reprodução do presente estudo, em outras regiões do Brasil, seria de grande valia para a realização de um mapeamento de fatores positivos e negativos de aspectos físicos da QV de adolescentes, bem como identificação de subgrupos populacionais com risco de apresentar QV ruim.

Em conclusão, os principais subgrupos populacionais com risco de apresentar domínio físico ruim foram adolescentes que apresentaram NAF baixo e pertencentes ao sexo feminino. Com base nestes achados, parece ser necessário que estudos futuros de intervenção consideremo NAF e o sexo no planejamento e desenvolvimento de programas de promoção à saúde adolescente e melhoria da QV •

\section{REFERÊNCIAS}

1. Andersen LB, Harro M, Sardinha LB, Froberg K, Ekelund U, Brage S, et al. Physical activity and clustered cardiovascular risk in children: a cross-sectional study (The European Youth Heart Study). Lancet 2006; 368(9532): 299-304.

2. Carneiro G, Ribeiro Filho FF, Togeiro SM, Tufik S, Zanella MT. Interações entre síndrome da apnéia obstrutiva do sono e resistência à insulina. Arq Bras Endocrinol Metabol 2007; 51(7): 1035-1040.

3. Organização Pan-americana da Saúde [Internet]. Doenças crônico-degenerativas e a obesidade: estratégia mundial sobre alimentação saudável, atividade física e saúde; 2003. Disponível em: http://www.opas.org.br/sistema/arquivos/d cronic.pdf. Acesso junho de 2007.

4. Brown DW, Balluz LS, Heath GW, Moriarty DG, Ford ES, Giles WH, et al. Associations between recommended levels of physical activity and health-related quality of life findings from the 2001 Behavioral Risk Factor Surveillance System (BRFSS) survey. Prev Med 2003; 37(5): 520-528.

5. Huang IC, Frangakis C, Wu AW. The relationship of excess body weight and health-related quality of life: evidence from a population study in Taiwan. Int J Obes 2006; 30(8):1250-1259.

6. Carlini-Cotrim BC, Gazal-Carvalho C, Gouveia N. Comportamentos de saúde entre jovens estudantes das redes pública e privada da área metropolitana do Estado de São Paulo. Rev Saúde Pública 2000; 34(6): 636-645. 
7. Hanson MD, Chen E. Socioeconomic status, race, and body mass index: The mediating role of physical activity and sedentary behaviors during adolescence. J Pediatr Psychol 2007; 32(3): 250-259.

8. Yamamoto-Kimura L, Posadas-Romero C, Posadas-Sánchez R, Zamora-González J, Cardoso-Saldaña G Ramírez IM. Prevalence and interrelations of cardiovascular risk factors in urban and rural Mexican adolescents. J Adolesc Health 2006; 38(5): 591-598.

9. Gil AC. Métodos e técnicas de pesquisa social. 3 Edição. São Paulo: Atlas; 1991.

10. The Whogol Group [editorial]. The World Health Organization quality of life assessment (WHOQOL): development and general psychometric properties. Soc Sci Med 1998; 46(12): 1569-1585.

11. Fleck MPA, Louzada S, Xavier M, Chachamovich E, Vieira G, Santos L, et al. Aplicação da versão em português do instrumento abreviado de avaliação da qualidade de vida "WHOQOL-bref". Rev Saúde Pública 2000; 34(2): 178-183.

12. Izutsu T, Tsutsumi A, Islam A, Matsuo Y, Yamada HS, Kurita H, et al. Validity and reliability of the Bangla version of WHOQOL-BREF on an adolescent population in Bangladesh. Qual Life Res 2005; 14(7): 1783-1789.

13. The Australian Centre for Posttraumatic Mental Health [Internet]. Trauma related research, training and policy development, 2003. Disponível em: http://www.psychiatry.unimelb.edu.au/ qo/whogol/ whogol-insruments.html\#algorithms. Acesso abril de 2006.

14. Guedes DP, Lopes CC, Guedes JERP. Reprodutibilidade e validade do Questionário Internacional de Atividade Física em adolescentes. Rev Bras Med Esporte 2005; 11(2): 151-158.

15. Lima CT, Freire ACC, Silva APB, Teixeira RM, Farrell M, Prince M. Concurrent and construct validity of the audit in an urban Brazilian sample. Alcohol Alcohol 2005; 40(6): 584-589.

16. BaborTF, Higgins-BiddleJC, Saunders JC, Monteiro MG AUDIT-TheAlcohol UseDisorders Identification Test: Guidelines for Use in Primary Health Care. WHO/MSD/MSB/01.6a, World Health Organization, Geneva; 2001.

17. Gordon CC, Chumlea WCC, Roche AF. Stature, recumbent length, and weight. In: Lohman TG Roche AF, Martorell R, eds. Anthropometric standardization reference manual. Champaign: Human Kinetics; 1988

18. Conde WL, Monteiro CA. Valores críticos do índice de massa corporal para classificação do estado nutricional de crianças e adolescentes brasileiros. J Pediatr 2006; 82(4): 266-272.

19. Organização Mundial da Saúde [editorial]. Who Expert Committee on Physical Status: The use and interpretation of anthropometry physical status. Geneva: World Health Organization; (WHO Technical Report Series, v. 854); 1995.

20. Associação Brasileira de Empresas de Pesquisa [Internet]. Dados com base no Levantamento Sócio Econômico, 2000-IBOPE. Disponível em: http:/www.abep.org. Acesso maio de 2005.

21. Vilela Junior GB, Gordia AP, Quadros TMB, Campos W. Relation between quality of life and alcohol consumption for students attending the physical education and sports course. FIEP Bulletin 2007; 77: 231-235.

22. Izutsu T, Tsutsumi A, Islam AM, Kato S, Wakai S, Kurita H. Mental health, quality of life, and nutritional status of adolescents in Dhaka, Bangladesh: comparison between an urban slum and a nonslum area. Soc Sci Med 2006; 63(6): 1477-1488.

23. Lipp MEN, Tanganelli MS. Stress e qualidade de vida em Magistrados da Justiça do Trabalho: diferenças entre homens e mulheres. Psicol Reflex Crit 2002; 15(3): 537-548.

24. Cucchiaro G Dalgalarrondo P. Saúde mental e qualidade de vida em adolescentes: um estudo entre escolares em duas áreas urbanas contrastantes. Rev Bras Psiquiatr 2007; 29(3): 213-221.

25. Chen X, Sekine M, Hamanishi S, Yamagami T, Kagamimori S. Associations of lifestyle factors with quality of life (QOL) in Japanese children: a 3-year follow-up of the Toyama Birth Cohort Study. Child Care Health Dev 2005; 31(4): 433-439.

26. Wang $\mathrm{H}$, Sekine M, Chen X, Yamagami T, Kagamimori S. Lifestyle at 3 years of age and quality of life (QOL) in first-year junior high school students in Japan: results of the Toyama Birth Cohort Study. Qual Life Res 2008; 17(2): 257-265.

27. Hallal PC, Dumith SC, Bastos JP, Reichert FF, Siqueira FV, Azevedo MR. Evolução da pesquisa epidemiológica em atividade física no Brasil: revisão sistemática. Rev Saúde Pública 2007; 41(3): 453-460. 